\title{
Commentary \\ Clinical risk stratification for gastrointestinal hemorrhage: still no consensus
}

\author{
Charles Wira ${ }^{1}$ and John Sather ${ }^{2}$
}

\begin{abstract}
1Department of Surgery, Section of Emergency Medicine, Yale School of Medicine, 464 Congress Ave., Suite 260, New Haven, CT 06519, USA 2Department of Surgery, Section of Emergency Medicine and Surgical Critical Care, Yale School of Medicine, 464 Congress Ave., Suite 260, New Haven, CT 06519, USA
\end{abstract}

Corresponding author: Charles Wira, Charles.wira@yale.edu

Published: 30 May 2008

This article is online at http://ccforum.com/content/12/3/154

(c) 2008 BioMed Central Ltd

See related research by Das et al., http://ccforum.com/content/12/2/R57
Critical Care 2008, 12:154 (doi:10.1186/cc6900)

diverse population [6]. Early endoscopy is often impractical in the Emergency Department, thus necessitating the promulgation of sensitive clinical variables to determine illness severity. Prognostic factors indicative of hemodynamic stabilization or decompensation have been evaluated in patients with a presumed upper GIH $[10,11]$ or a presumed lower GIH $[12,13]$.

Kollef and colleagues, in the original BLEED study, classified patients presenting with $\mathrm{GIH}$ as at high risk to develop significantly greater rates of inhospital complications if they had bleeding, hypotension, an elevated prothrombin time, or erratic mental status [2]. Afessa found an independent association of hepatic cirrhosis, high Acute Physiologic and Chronic Health Evaluation II scores, active GIH, and endorgan dysfunction with similar complications [8]. Inayet and colleagues identified a correlation between ICU admission and an elevated prothrombin time, hypotension, Acute Physiologic and Chronic Health Evaluation II score $>15$, and acute neurologic change [7]. They reported a sensitivity of $88 \%$ and a specificity of $74 \%$ for subsequent instability. Their study highlighted the importance of identifying patients who would not just bleed, need surgery, or die, but those patients who would actually warrant hemodynamic stabilization in an ICU.

Das and colleagues' scientific questions in their manuscript are therefore important [1]. The design was a derivation and validation study testing the original BLEED criteria, with the additional development of a triage simulation model. The authors recognized many of the challenges facing Emergency Department providers and designed their study to incorporate objective data routinely available in the Emergency Department. They also included patients with either upper

BLEED criteria = ongoing bleeding, low systolic blood pressure, elevated prothrombin time, erratic mental status, unstable comorbid disease; GIH = gastrointestinal hemorrhage; ICU = intensive care unit. 


\section{Table 1}

\begin{tabular}{ll}
$\begin{array}{l}\text { Pre-endoscopic variables that may risk-stratify patients with } \\
\text { gastrointestional hemorrhage }\end{array}$ \\
\hline B & Base-deficit abnormal \\
A & Antiplatelet or Anticoagulation agents being taken by patient \\
D & Decrease in serial hematocrit measurements \\
U & Urine output impaired \\
P & Presyncope or syncope \\
P & Postural hypotension \\
E & Electrocardiogram with ischemic changes \\
R & Reduced central venous pressure (ultrasound or via catheter) \\
& \\
L & Lactic acidosis \\
O & Organ failure \\
W & Low wedge pressure (echocardiogram or via catheter) \\
E & Elevated shock index \\
R & Racing tachycardic heart \\
& \\
G & Geriatric patient \\
I & Strong ion difference \\
& \\
B & Ongoing bleeding \\
L & Low blood pressure \\
E & Elevated coagulation factors \\
E & Erratic mental status \\
D & Comorbid disease \\
\hline
\end{tabular}

Adapted from Kollef and colleagues [2].

GIH or lower GIH, also of value to Emergency Department providers since in an acute presentation the culprit lesion may be unknown in almost $20 \%$ of patients $[2,8]$.

Das and colleagues' data suggest that visible signs of ongoing bleeding or an elevated prothrombin time may be associated with their defined complications of death or rebleeding. Although limited by a small sample size, their study serves to externally validate components of the prior Kollef and colleagues' trial [2]. Like prior literature [7], however, their reported sensitivity of $73 \%$ to $83 \%$ to rule out complications in GIH ideally needs to be higher.

Prior to the generation of consensus guidelines using preendoscopic variables to determine whether patients need ICU monitoring, overall sensitivity in the cumulative existing literature needs to be improved in order to ensure that patients triaged to routine medical floors will not hemodynamically decompensate. Designing an appropriate study to establish clinical triage criteria for patients with upper GIH or lower GIH is challenging. Ideally a derivation and validation study would need to be appropriately powered, multicentered, and implemented in either a randomized or before-after design, with a gold standard of early endoscopy, clinical variables, and short-term outcome. Das and colleagues are to be commended for extending their analysis to look at other variables (that is, the shock index), and future investigation should more broadly encompass other clinical variables (Table 1) that have been utilized in other settings to discern bleeding patients at risk for escalation of care (that is, the trauma and cerebral hemorrhage literature) $[14,15]$.

Das and colleagues are therefore to be applauded for their publication's contribution to the growing number of studies evaluating clinical and pre-endoscopic factors risk-stratifying patients with $\mathrm{GIH}$. Further investigation, however - either from future studies or from the pooling of investigator databases - needs to comprehensively look at all clinical variables involved in the GIH triage process in order to more accurately, and with higher sensitivity, determine who needs ICU monitoring prior to endoscopy.

\section{Competing interests}

The authors declare that they have no competing interests.

\section{Acknowledgement}

The authors wish to thank Sharon Fontecchio, BSN, for her support in this publication.

\section{References}

1. Das AM, Sood N, Hodgin K, Chang L, Carson SS: Development of a triage protocol for patients presenting with gastrointestional hemorrhage: a prospective cohort study. Crit Care 2008, 12:R57.

2. Kollef MF, O'Brien JD, Zuckerman GR, Shannon W: BLEED: a classification tool to predict outcomes in patients with acute upper and lower gastrointestinal hemorrhage. Crit Care Med 1997, 25:1125-1132.

3. Longstreth GF: Epidemiology of hospitalization for acute upper gastrointestinal hemorrhage: a population-based study. Am J Gastroenterol 1995, 90:206-210.

4. Longstreth GF: Epidemiology and outcome of patients hospitalized with acute lower gastrointestinal hemorrhage: a population-based study. Am J Gastroenterol 1997, 92:419-424.

5. Peura DA, Lanza FL, Gostout CJ, Foutch PG: The American College of Gastroenterology Bleeding Registry: preliminary findings. Am J Gastroentero/ 1997, 92:924-928.

6. Elmunzer BJ, Inadomie JM, Elta GH: Risk stratification in upper gastrointestional bleeding. J Clin Gastroenterol 2007, 41:559563.

7. Inayet N, Amoateng-Adjepong Y, Upadya A, Manthous CA: Risks for developing critical illness with Gl hemorrhage. Chest 2000, 118:473-478.

8. Afessa B: Triage of patients with acute gastrointestinal bleeding for intensive care unit admission based on risk factors for poor outcome. J Clin Gastroentero/ 2000, 30:281-285.

9. Farrell RJ, Alsahli M, LaMont JT: Is successful triage of patients with upper-gastrointestional bleeding possible without endoscopy? Lancet 2000, 356:1 289-1290.

10. Adamopoulos AB, Baibas NM, Efstathiou SP, Tsioulos DI, Mitromaras AG, Tsami AA, Mountokalakis TD: Differentiation between patients with acute upper gastrointestinal bleeding who need early urgent upper gastrointestinal endoscopy and those who do not. A prospective study. Eur J Gastroenterol Hepatol 2003, 15:381-387.

11. Blatchford O, Murray WR, Blatchford M: A risk score to predict need for treatment for upper-gastrointestinal haemorrhage. Lancet 2000, 356:1318-1321.

12. Strate LL, Saltzman JR, Ookubo R, Mutinga ML, Syngal S: Validation of a clinical prediction rule for severe acute lower intestinal bleeding. Am J Gastroentero/ 2005, 100:821-827. 
13. Velayos FS, Williamson A, Sousa KH, Lung E, Bostrom A, Weber EJ, Ostroff JW, Terdiman JP: Early predictors of severe lower gastrointestinal bleeding and adverse outcomes: a prospective study. Clin Gastroenterol Hepatol 2004, 2:485-490.

14. Hoyt DB, Coimbra R: Trauma systems. Surg Clin N Am 2007, 87:21-35.

15. Kaplan $L$, Kellum JA: Comparison of acid base models for prediction of mortality following trauma. Shock 2007, in press [Epub ahead of print]. 